\title{
Interdisciplinary Research Funding Mechanisms of the UK Research and Innovation (UKRI) and Their Implications
}

\author{
Wencong $\mathrm{Li}^{1}{ }^{1}{ }^{\star}$, Jin Xu ${ }^{1}$, Jie Shen ${ }^{1}$, Juanjuan Liu ${ }^{2}$, Yingjie Fan ${ }^{1}$, Junlin Yang ${ }^{1}$ \\ ${ }^{1}$ Bureau of International Cooperation, National Natural Science Foundation of China, Beijing 100085, P. R. China. \\ ${ }^{2}$ Cuiying Biomedical Research Center, Lanzhou University Second Hospital, Lanzhou 730030, P. R. China.
}

\begin{abstract}
Newly established in 2018, the UK Research and Innovation (UKRI) strengthens the strategic coordination of the UK research and innovation system by bringing together seven Research Councils, Research England, and Innovate UK. Through its nine organizations, UKRI funds multidisciplinary and interdisciplinary research in a number of priority areas. It also runs the Strategic Priorities Fund to support multidisciplinary and interdisciplinary research in strategic areas identified by government policies as well as the Global Challenges Research Fund to promote challenge-led interdisciplinary research needed by developing countries. The UKRI makes significant efforts to engage stakeholders in the development, design, and implementation of multidisciplinary and interdisciplinary programs. It has also developed a range of mechanisms to improve the evaluation of multidisciplinary and interdisciplinary projects. Chinese science and innovation funding agencies could draw upon the UKRI experience from four aspects to advance interdisciplinary research in China.
\end{abstract}

Key Words: Interdisciplinary research; Funding mechanism; Organizational structure; Program management; Peer review

\section{英国国家科研与创新署学科交叉研究资助机制及启示}

\author{
李文聪 ${ }^{1, *}$, 徐进 ${ }^{1}$, 申洁 ${ }^{1}$, 刘娟娟 ${ }^{2}$, 范英杰 ${ }^{1}$, 杨俊林 ${ }^{1}$ \\ 1 国家自然科学基金委员会国际合作局，北京 100085 \\ 2 兰州大学第二医院萃英生物医学研究中心, 兰州 730030
}

\begin{abstract}
摘要: 自2018年成立以来, 英国国家科研与创新署(UKRI)整合了以学科为基础划分的英国七大研究理事会和英国创新 署、英格兰研究署, 显著增强了英国科研与创新体系的战略协同。通过九个下属机构的横向联合, UKRI在一批重点领域 资助了多个学科交叉项目。此外, UKRI还通过战略优先基金支持面向国家战略需求的学科交叉研究, 通过全球挑战研究 基金促进以学科交叉手段解决发展中国家面临的共性挑战。在学科交叉项目的立项和管理过程中, UKRI重视与政府、大 学、非政府组织以及产业界的合作, 并采取一系列手段改善学科交叉项目的评审效果。UKRI的举措对我国科研创新资助 机构推动学科交叉发展具有多方面的借鉴意义, 如优化资助格局、完善跨学科跨部门协作机制、加强与政府和企业的合 作、以及改进评审机制等。
\end{abstract}

关键词：学科交叉; 资助机制; 组织结构; 项目管理；同行评议 中图分类号: G463; G311

\section{1 引言}

经济与科技的迅猛发展与全球性挑战的不断

涌现, 要求打破学科之间的条块分割, 促进学科交 叉融合。多国政府和科研资助机构日益重视学科

Received: August 18,2020; Revised: August 18, 2020; Accepted: August 18, 2020; Published online: August 26, 2020.

*Corresponding author. Email: liwc@nsfc.gov.cn; Tel.: +86-10-62327014.

(C) Editorial office of Acta Physico-Chimica Sinica 
交叉融合的重要性, 采取多种手段推进学科交叉 研究。英国的研究理事会(Research Councils)是以 研究项目或研究计划的形式支持大学和公共研究 机构开展科学研究的资助机构, 项目分配采取同 行评议竞争机制, 经费来自政府投入。以学科为基 础划分设有艺术及人文科学研究理事会(AHRC)、 生物技术与生物科学研究理事会(BBSRC)、工程 与自然科学研究理事会(EPSRC)、经济与社会科学 研究理事会(ESRC)、医学研究理事会(MRC)、自然 环境研究理事会(NERC)、科学及技术设施理事会 (STFC)等 7 个独立的研究理事会。2018 年, 英国 政府整合上述 7 个研究理事会与英国创新署 (IUK)、英格兰研究署(Research England), 组建英 国国家科研与创新署 (以下简称 UKRI), 意在加强 宏观统筹、整合资源, 将各个学科以及从基础研究 到商业创新的完整链条纳入统一的资助框架, 提 高创新投入与产出效率。UKRI 赋予多/跨学科、跨 部门研究项目重要的战略定位, 其对学科交叉研 究的资助机制对我国科研与创新资助机构具有丰 富的借鉴意义。

\section{2 英国国家科研与创新署的组织结构}

UKRI 成立后, 其下属 9 个机构依然是根据 《高等教育和研究法》以及皇家宪章成立的独立 法人实体 ${ }^{1}$, 负责制定各自所涉及学科领域的资助 政策以及具体项目管理工作, 在组织结构上均设
有 1 名执行主席和一个由 5-12 名成员组成的委员 会。这使得 9 个机构依然享有充分的独立性、灵 活性和创造性, 从而保证对不同学科、不同部门的 资助特色和资助力度。为了从更高层次上发挥战 略引领作用, 高效地协调各下属机构的工作, UKRI 在组织结构上设立了董事会、执行委员会和业务 服务中心(图 1)。

董事会包括 1 名主席、UKRI 首席执行官、 UKRI 首席财务官和 9-12 名来自大学、研究机构 和企业界的董事。他们均由英国商务、能源与产业 战略部(BEIS)国务大臣任命并向其汇报工作, 任期 3-5 年。董事会的职责是制定 UKRI 的战略、政策 与规划, 并监督 UKRI 的运营和资金使用是否符 合董事会制定的战略目标。董事会下设审计、风 险、认证与绩效委员会和提名与薪酬委员会等两 个内部监督委员会, 分别从监督审计和人事两个 方面支持董事会发挥领导作用。

执行委员会负责向董事会提出战略与政策建 议, 也是把 UKRI 战略与政策推向贯彻落实的协 调机构。执行委员会由 UKRI 首席执行官主持工 作，其他成员包括 UKRI 首席财务官、9个下属机 构的执行主席等, 下设人力财务和运营委员会、战 略委员会和投资委员会。这种设计既使 UKRI 能 够从战略和运营层面推进各下属机构之间的协调 与合作，也保证了 UKRI 董事会制定的政策方针 能够通过 9 位执行主席体现在各下属机构自身的

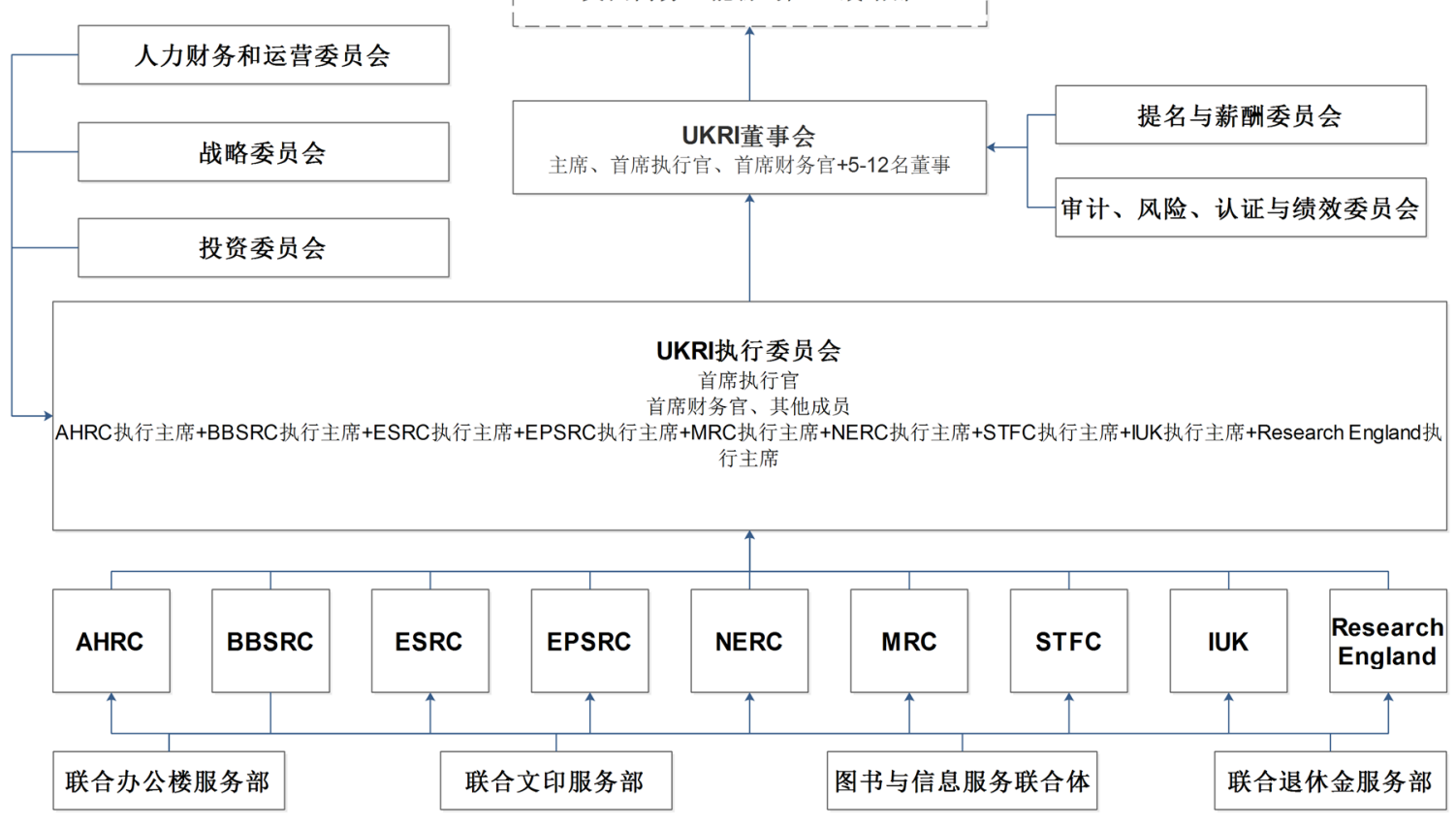

图 1 英国国家科研与创新署组织结构图

Fig. 1 Organizational structure of UKRI. 
政策和行动中。

业务服务中心是为各下属机构提供共性行政 服务的机构, 包括联合办公楼服务部、联合文印服 务部、联合退休金服务部和图书与信息服务联合 体。它们将一般性行政事务从各下属机构业务中 剥离出来, 有利于降低行政成本, 使各下属机构更 加专注于业务工作。

综上所述, UKRI 在组织结构上的特色在于保 留了各下属机构的独立性, 同时又从制定战略规 划、推动横向联合、开展监督评估以及综合行政服 务等几个方面充分发挥引领、督导和集成作用。这 一理念也体现在其对学科交叉研究的资助机制 中。

\section{3 英国国家科研与创新署的学科交叉研究 项目资助体系}

英国各研究理事会之间通过横向联合开展学 科交叉研究资助已经有约 20 年的历史。UKRI 成 立后, 多/跨学科项目仍然主要由各下属机构联合 实施。此外, 在 UKRI 现有项目类型中, 战略优先 基金 (SPF) 和全球挑战研究基金(GCRF)也具有明 显的学科交叉特色。

\section{1 多/跨学科项目}

多/跨学科项目通常由某个下属机构牵头, 多 个下属机构参与, 也可以联合英国政府部门、地方 政府部门、其他研究机构和私人基金会提供政策、 资金等方面的支持。当前正在实施的多/跨学科项 目有 7 个, 主题包括数字经济、能源、全球食品安 全、抗生素耐药、生命科学研究新技术、动植物健 康以及城市生活(表 1)。其中数字经济、能源和全 球食品安全等 3 个项目在 UKRI 成立前已实施多 年。例如能源项目起源于 2002 至 2005 年期间由
EPSRC 牵头实施的 “可持续能源经济” 项目, 是 研究理事会联合资助学科交叉项目的最早尝试之

\section{2 战略优先基金}

战略优先基金设立于2018年，起因是2015年 诺贝尔奖得主、英国皇家学会前主席保罗·纳斯爵 士在对各研究理事会进行评估后提出, 应当设立 一个独立于各研究理事会之外的基金, 专门支持 传统资助渠道无法有效涵盖的多学科及跨学科科 研与创新项目 ${ }^{2}$ 。战略优先基金的经费来源是英国 财政部 “国家生产力投资基金” (NPIF)，投入总 额达 8.3 亿英镑, 是英国公共财政经费支持力度最 大的学科交叉基金。战略优先基金具有三大目标: 推动高质量多学科及跨学科研究和创新、确保政 府资金投入紧紧围绕研究与创新重点和当前机 遇, 确保科研体系面向战略性领域和挑战。

战略优先基金采用 “竞争择优” 的方式遴选 产生优先领域。UKRI 各下属机构以及英国国家物 理实验室、英国原子能管理局等其他 6 家受英国 商务、能源与产业战略部资助的机构可以向立项 评审委员会提交立项申请。立项评审委员会负责 评选立项计划, 并确定资助预算与强度。委员会现 有 10 名成员，包括 UKRI 首席执行官、董事会成 员、首席财务官、战略委员会主任以及英国政府首 席科学顾问、英国商务能源与产业战略部官员及 英国大学代表等。评审委员会的组成体现了 UKRI 与政府科技办公室和内阁部门的密切合作, 有利 于战略优先基金与政府科技规划的结合。目前, 战 略优先基金已完成 2 轮立项评审, 遴选并立项了 34 个学科交叉项目(表 2)。在领域选择上, 战略优 先基金更青睐与产业核心竞争力、国家安全以及 国计民生有关的科学问题, 很多项目与政府政策

表 1 UKRI 多/跨学科项目

Table 1 Multidisciplinary/interdisciplinary programs of UKRI.

\begin{tabular}{cl}
\hline 主题 & \\
\hline 数字经济 & EPSRC, AHRC, ESRC, IUK \\
能源 & EPSRC, BBSRC, ESRC, NERC, STFC \\
全球食品安全 & BSRC, ESRC, EPSRC, MRC, NERC, STFC, 商业能源与产业战略部, 环境食品与农村事务部, 国际贸易部, 卫 \\
& 生部, 国际发展部, 食品标准署, 政府科学办公室, 英国气象局, 地方政府部门, 惠康基金会 \\
抗生素耐药 & MRC, AHRC, BBSRC, ESRC, EPSRC, IUK, NERC, STFC, 商业能源与产业战略部, 国际开发部, 国防科技实 \\
& 验室, 环境食品与农村事务部, 卫生部, 食品标准署, 地方政府部门, 惠康基金会 \\
生命科学研究新技术 & BBSRC, EPSRC, MRC \\
动植物健康 & BBSRC, IUK, 环境食品与农村事务部, 政府科学办公室, 地方政府部门, 国际开发部, 商业能源与产业战略部 \\
城市生活 & AHRC, BBSRC, EPSRC, ESRC, MRC, NERC, STFC, IUK \\
\hline
\end{tabular}


表 2 战略优先基金

Table 2 Strategic priorities fund.

\begin{tabular}{|c|c|c|c|}
\hline 立项年份 & 主题 & 项目名称 & 经费(百万英镑) \\
\hline \multirow[t]{4}{*}{2018} & 环境 & 清洁空气: 分析与解决方案 & 19.6 \\
\hline & & 气候变化恢复力 & 18.7 \\
\hline & & 建设数字化环境 & 10.4 \\
\hline & & 提升土地利用决策水平 & 10.5 \\
\hline \multirow[t]{4}{*}{2018} & 生物与生物医学 & 生命的物理学 & 31.2 \\
\hline & & 生命科学数据资源 & 45.0 \\
\hline & & 微生物造成的植物疾病 & 17.7 \\
\hline & & 人类细胞图谱 & 6.7 \\
\hline \multirow[t]{3}{*}{2018} & 人工智能 & 机械化与生活 & 9.2 \\
\hline & & 人工智能与数据科学 & 38.8 \\
\hline & & 数字技术(物联网)安全性 & 30 \\
\hline \multirow[t]{3}{*}{2018} & 生产力 & 英国人口实验室 & 2.1 \\
\hline & & 生产力提升研究 & 8.9 \\
\hline & & 面向企业产品、服务和生产过程的分析服务 & 14.1 \\
\hline 2018 & 基础设施 & 极限光子学应用中心 & 81 \\
\hline \multirow[t]{5}{*}{2019} & 环境 & 温室气体减排示范 & 31.52 \\
\hline & & 清洁空气: 室内与室外交界面 & 22.0 \\
\hline & & 以国民健康与健康环境为目标的食品体系转型 & 47.5 \\
\hline & & 循环经济跨学科研究项目 & 30 \\
\hline & & 海洋资源可持续管理 & 12.4 \\
\hline \multirow[t]{6}{*}{2019} & 健康、福利与人权 & 现代奴役制与人权的政策和证据研究中心 & 10.0 \\
\hline & & 核酸疗法加速器 & 30.0 \\
\hline & & 高级疼痛发现平台：探索慢性疼痛的复杂性 & 24.0 \\
\hline & & 成人社会护理循证实施研究中心 & 15.0 \\
\hline & & 大规模处理多病共患问题 & 20.0 \\
\hline & & 青春期、心理健康与心智发展 & 35.0 \\
\hline \multirow[t]{2}{*}{2019} & 数字技术 & 向世界开放(数字化)英国文化遗产 & 19.0 \\
\hline & & 保护公民网络个人隐私 & 18.2 \\
\hline \multirow[t]{6}{*}{2019} & 生产力与技术 & 空间天气、创新、测量、建模和风险 & 20.0 \\
\hline & & 对英国生产力挑战的系统研究 & 42.2 \\
\hline & & 可信赖的自动化系统 & 34.0 \\
\hline & & 增强超级计算 & 47.5 \\
\hline & & 国家卫星时统中心 & 36.0 \\
\hline & & 面向基础物理学的量子技术 & 40.0 \\
\hline
\end{tabular}

数据来源: UKRI 网站 https://www.ukri.org/research/themes-and-programmes/strategic-priorities-fund/

紧密结合, 并得到了多个政府部门的密切参与和 政策支持。

\section{3 全球挑战研究基金}

全球挑战基金是英国政府用开发援助资金 (ODA)设立的前沿研究基金,面向发展中国家面临 的共性挑战，总投入约 15 亿英镑。UKRI 是该基 金的 9 所实施机构之一。全球挑战基金是以国际
合作为特色的学科交叉基金, 不仅支持英国研究 人员与发展中国家研究人员开展合作，还与发展 中国家的科研创新资助机构共同资助双多边国际 合作项目。全球挑战基金的主题与联合国可持续 发展目标(SDGs)具有高度的一致性, 共分为三大 板块, 一是公平的可持续发展机会, 包括食品、健 康与幸福、公平教育、清洁空气水源与卫生设施、 
能源等研究方向; 二是可持续经济与社会发展, 包 括生活方式、环境变化、城市发展、生产与消费等 研究方向; 三是人权、治理与正义，包括难民危机、 地区冲突、减贫与消除不平等等研究方向。

为了突出对跨学科研究的支持, 全球挑战基 金征集并资助了 12 个非实体跨学科研究中心 (Interdisciplinary Research Hubs)项目, 总投资超过 2 亿英镑(表 3), 吸纳了来自 85 个国家的 550 余名 科研人员。

\section{4 英国国家科研与创新署学科交叉项目的 立项与管理}

UKRI 学科交叉项目涉及多个下属机构以及 政府部门和外部机构, 其立项和管理过程通常遵 循以下步骤: 首先, 具有共同兴趣的下属机构和合 作机构成立一个跨部门协调小组, 对项目进行调 研和论证, 撰写战略规划报告。然后, 小组向决策 部门报告并申请预算, 经费预算可来自各下属机 构自有预算, 也可以申请政府的特殊基金, 例如 BBSRC 等 “全球食品安全” 项目的实施单位向 战略优先基金申请经费, 设立了 “以国民健康与 健康环境为目标的食品体系转型” 项目, 这个项 目同时也是 “全球食品安全” 项目的一部分。预 算得到批准后, 在撰写具体项目指南的过程中, 相 关 UKRI 下属机构和合作机构还要组织召开一系 列研讨会, 广泛邀请多学科专家学者、政府官员、 企业人士以及社会工作者共同讨论具体的资助领

\section{表3 全球挑战基金跨学科研究中心}

\section{Table 3 Global challenges research fund global}

Interdisciplinary Research Hubs.

\begin{tabular}{cc}
\hline 名称 & 经费(百万英镑) \\
\hline 促进非洲青少年成长研究中心 & 18.5 \\
城市棚户区公平性研究中心 & 12.1 \\
消除儿童发育障碍研究中心 & 18.2 \\
性别、正义与安全研究中心 & 15.2 \\
三角洲地带研究中心 & 15.3 \\
健康家禽研究中心 & 18.1 \\
海洋保护与可持续利用研究中心 & 18.2 \\
南亚氮循环研究中心 & 17.5 \\
南-南人口流动、不平等与发展研究中心 & 18.8 \\
贸易、发展和环境研究中心 & 18.2 \\
城市灾害风险研究中心 & 17.6 \\
水安全与可持续发展研究中心 & 17.7 \\
\hline
\end{tabular}

数据来源: UKRI网站https://www.ukri.org/files/news/ukri-gcrf-globalinterdisciplinary-research-hubs/
域。最后发布项目指南前, 通常会确定由某个下属 机构承担受理申请和组织评审等具体工作, 其他 相关下属机构则参与推荐评审专家。

UKRI 学科交叉项目的管理结构通常包括 “项目协调小组”、“战略咨询委员会” 和 “科 学顾问小组” 等 3 个部分。以“全球食品安全” 项目为例 ${ }^{3}$ (图 2), 其项目协调小组包含所有参与 该项目的实施机构代表(每个机构在小组中有 1 名 代表), 负责协调各项活动以及与其他食品安全项 目的联络(例如战略优先基金), 监督具体工作的实 施, 由各实施机构轮流主持工作。战略咨询委员会 负责对项目的战略导向提供建议, 确保全球食品 安全项目的资助重点符合政府政策与产业需求, 由政府、大学、非政府组织以及农业、食品、零售 企业代表组成, 其中包括英国政府首席科学顾问。 科学顾问小组由大学和研究机构的知名科学家组 成, 负责为全球食品安全项目提供学术意见, 确保 资助项目是最前沿的科学研究。

\section{5 英国国家科研与创新署学科交叉项目的 评审}

与单一学科项目的评审相比, 跨学科项目在 评审中面临多方面的不利因素, 突出的难点在于 遴选具备跨学科背景的同行评审专家 4 。UKRI 学 科交叉项目的评审过程包括通讯评审和会议评审 两个阶段, 针对跨学科项目的评审难点, UKRI 尤 其重视会议评审的作用。

在通讯评审阶段, 每份申请将发送给 3-6 名 具有不同学科背景的专家, 由他们打分并给出书 面评议。UKRI 工作人员负责收集评审专家在评议 中提出的具体问题并反馈给项目申请人, 要求项 目申请人在规定时间内提交书面答辩。书面答辩 材料将和申请书、通讯评审意见一起提交评审委 员会进行会议评审。

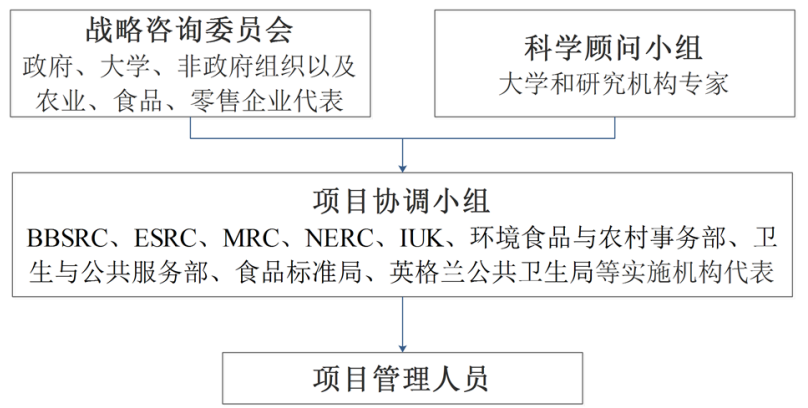

图 2 全球食品安全项目管理结构

Fig. 2 Governance structure of the global food security program. 
在会议评审中, UKRI 为每份申请分配至少 2 名不同学科背景的主审专家, 主审专家负责向评 审委员会全体成员介绍项目申请的基本情况，总 结通讯评审指出的问题, 点评申请人的书面答辩 是否合理回应了通讯评审专家的疑问，并各自给 出初始分数。主审专家发言后, 评审委员会全体成 员都要参加讨论，在形成共识的基础上共同确定 每个申请的最终得分。此外, UKRI 还会邀请一名 专业工作出色、管理经验丰富的学者担任评审会 主席, 主席不担任主审专家, 而是专门负责把控评 审进程和讨论方向, 引导具有多元背景的评审专 家从不同角度充分发表意见并最终形成共识。

对于与国家需求和产业发展密切相关的多/跨 学科项目, UKRI 还会在同行评审之外增加战略评 价环节，例如战略优先基金“循环经济跨学科研 究”项目的评审过程中, 由英国环境、食品和农村 事务部代表政府部门，对项目申请是否符合国家 战略和目标进行书面评估。值得指出的是, UKRI 在会议评审前向项目申请人反馈该评估的结果, 要 求项目申请人以书面形式进行回应, 作为评审材 料的一部分提交会议评审，供评审委员会参考 5 。

\section{6 英国国家科研与创新署的举措对我国发 展交叉学科和建立相应机制的借鉴意义}

自成立以来, UKRI 通过组织结构改革, 增强 了对下属机构的战略引领和下属机构之间的政策 协同。战略优先基金和全球挑战基金的设立，使 UKRI 学科交叉研究项目更加丰富, 进一步促进了 科学与技术以及经济社会发展的联系。目前, UKRI 以各下属机构对自身所属学科的资助工作 为 “经” , 以多/跨学科项目等跨理事会资助工作 为 “纬”, 构建了一个既支持各学科纵向深入发 展, 又促进学科之间交叉与融合的资助体系。 UKRI 的举措对我国科研与创新资助机构推动学 科交叉研究具有四个方面的借鉴意义:

一是优化资助格局, 突出战略需求。学科交叉 的推动力既有科学自身发展的内在需求, 又有经 济与社会发展的外在需求。资助机构应积极激发 外部动力, 加强科学界与其他部门的合作, 例如与 企业设立联合基金，并且在确定国际合作项目的 领域时更加突出学科交叉, 促使科研人员关注经 济社会发展中涌现的复杂问题和全球性挑战, 促
进科学技术为可持续发展提供支撑。

二是完善跨学科、跨部门协作机制。借鉴 UKRI 的经验, 可考虑从组织结构上着手, 设立专 责部门, 通过战略规划、项目实施统筹推进学科交 叉融合工作，从而加强顶层设计和不同学科领域 之间的协同。

三是在立项和管理过程中加强与政府、地方 和企业的合作。可通过组建战略咨询委员会以及 召开由多方代表参加的研讨会等方式, 充分吸纳 政府、大学、非政府组织以及产业界的意见，凝练 经济与社会发展需求背后具有战略性、关键性的 学科交叉研究领域及科学问题, 在项目实施过程 中定期开展评估, 确保科学研究与国家战略需求 的紧密结合。

四是改进跨学科项目评审机制, 重视发挥会 议评审的功能。长期实践证明, 会议评审对通讯评 审发挥着 “确认”、“纠偏” 和 “择优”功能, 而 会议评审的组织工作是关系到评审效果的关键 6 。 UKRI 通过选择多元学科背景评审专家、组织申请 人书面答辩、邀请资深学者引导讨论、全体专家在 共识基础上共同打分、引入政府参与评估等手段, 有效地促进了不同背景的评审人之间以及评审人 和申请人之间的交流。我国学科交叉项目评审机 制可考虑借鉴这些措施，以便对跨学科项目进行 全面的评价。

\section{References}

(1) https://www.gov.uk/government/publications/ukri-frameworkdocument/ (accessed on 29 May, 2020).

(2) https://www.gov.uk/government/publications/nurse-review-ofresearch-councils-recommendations (accessed on 29 May, 2020).

(3) https://www.foodsecurity.ac.uk/publications/global-food-securitystrategic-plan.pdf (accessed on 29 May, 2020).

(4) Wei, W.; Liu, F. F.; Liu, Z. L. Bull. Natl. Nat. Sci. Found. China 2016, 30 (6), 546. [魏巍, 刘凡丰, 刘仲林. 中国科学基金, 2016, 30 (6), 546.] doi: 10.16262/j.cnki.1000-8217.2016.06.016.

(5) https://epsrc.ukri.org/files/funding/calls/2020/ce-hub-call/ (accessed on 29 May, 2020).

(6) Zhang, G. J.; Fu, X. F.; Dai, Y. F.; Chen, Y. J. Acta Phys. -Chim. Sin 2020, 36 (8), 2003051. [张国俊, 付雪峰, 戴亚飞, 陈拥军. 物理化 学学报, 2020, 36 (8), 2003051.] doi: 10.3866/PKU.WHXB202003051 